\title{
5. AREA HEALTH SERVICES
}

Information on the health of Aboriginal and Torres Strait Islander mothers and mothers born in non-English speaking countries is shown in Chapters 7 and 8 respectively.

\section{Confinements}

The largest numbers of mothers who gave birth in 2006 were resident in the Sydney South West Area (20,426, 22.4 per cent), followed by the Sydney West Area (17,210, 18.8 per cent) and the South Eastern Sydney and Illawarra $(15,020,16.4$ per cent) Area (Table 20).

\section{Maternal age}

The proportion of women giving birth at less than 20 years of age varied from 1.6 per cent in the Northern Sydney and Central Coast Area to 8.9 per cent in the Greater Western Area, while the proportion of mothers giving birth at 35 years of age or more ranged from 13.1 per cent in the Greater Western Area to 31.4 per cent in the Northern Sydney and Central Coast Area (Table 20).

\section{Maternal country of birth}

Seventy-nine per cent of women who gave birth in NSW in 2006 were born in English speaking countries, 11.7 per cent were born in Asian countries, and 4.6 per cent were born in the Middle East or Africa (Table 21). The highest proportions of mothers born in non-English speaking countries were in the Sydney South West and Sydney West Areas. In the Sydney South West Area, the majority of mothers born in non-English speaking countries were born in South East Asia, and the Middle East and Africa. In the Sydney West Area, the most common maternal countries of birth were in the Middle East and Africa, and in Southern Asia.

\section{Maternal Aboriginality}

In 2006, 2.9 per cent of mothers were reported to be Aboriginal or Torres Strait Islander (Table 22). The proportion of Aboriginal or Torres Strait Islander mothers varied from 0.7 per cent in the Northern Sydney and Central Coast Area to 13.8 per cent in the Greater Western Area.

\section{TABLE 20}

MATERNAL AGE AND HEALTH AREA OF RESIDENCE, NSW 2006

\begin{tabular}{|c|c|c|c|c|c|c|c|c|c|c|c|c|c|c|c|c|c|c|}
\hline \multirow[t]{3}{*}{ Health Area } & \multicolumn{18}{|c|}{ Maternal age (years) } \\
\hline & \multicolumn{2}{|c|}{$12-19$} & \multicolumn{2}{|c|}{$20-24$} & \multicolumn{2}{|c|}{$25-29$} & \multicolumn{2}{|c|}{$30-34$} & \multicolumn{2}{|c|}{$35-39$} & \multicolumn{2}{|c|}{$40-44$} & \multicolumn{2}{|c|}{$45+$} & \multicolumn{2}{|c|}{ Not stated } & \multicolumn{2}{|c|}{ TOTAL } \\
\hline & No. & $\%$ & No. & $\%$ & No. & $\%$ & No. & $\%$ & No. & $\%$ & No. & $\%$ & No. & $\%$ & No. & $\%$ & No. & $\%$ \\
\hline Sydney South West & 602 & 2.9 & 2786 & 13.6 & 5729 & 28.0 & 6859 & 33.6 & 3707 & 18.1 & 702 & 3.4 & 34 & 0.2 & 7 & 0.0 & 20426 & 100.0 \\
\hline $\begin{array}{l}\text { South Eastern } \\
\text { Sydney and } \\
\text { Illawarra }\end{array}$ & 326 & 2.2 & 1388 & 9.2 & 3669 & 24.4 & 5769 & 38.4 & 3208 & 21.4 & 622 & 4.1 & 34 & 0.2 & 4 & 0.0 & 15020 & 100.0 \\
\hline Sydney West & 642 & 3.7 & 2739 & 15.9 & 5278 & 30.7 & 5519 & 32.1 & 2543 & 14.8 & 464 & 2.7 & 21 & 0.1 & 4 & 0.0 & 17210 & 100.0 \\
\hline $\begin{array}{l}\text { Northern Sydney } \\
\text { and Central Coast }\end{array}$ & 228 & 1.6 & 1044 & 7.4 & 2897 & 20.5 & 5533 & 39.1 & 3727 & 26.4 & 677 & 4.8 & 31 & 0.2 & 2 & 0.0 & 14139 & 100.0 \\
\hline $\begin{array}{l}\text { Hunter and New } \\
\text { England }\end{array}$ & 683 & 6.5 & 1893 & 17.9 & 3081 & 29.1 & 3181 & 30.1 & 1460 & 13.8 & 259 & 2.4 & 16 & 0.2 & 6 & 0.1 & 10579 & 100.0 \\
\hline North Coast & 361 & 7.1 & 1054 & 20.7 & 1368 & 26.8 & 1420 & 27.8 & 748 & 14.7 & 137 & 2.7 & 10 & 0.2 & 2 & 0.0 & 5100 & 100.0 \\
\hline Greater Southern & 246 & 6.1 & 837 & 20.6 & 1148 & 28.3 & 1134 & 27.9 & 580 & 14.3 & 106 & 2.6 & 8 & 0.2 & 0 & 0.0 & 4059 & 100.0 \\
\hline Greater Western & 359 & 8.9 & 836 & 20.6 & 1149 & 28.4 & 1151 & 28.4 & 438 & 10.8 & 85 & 2.1 & 7 & 0.2 & 27 & 0.7 & 4052 & 100.0 \\
\hline Other - not stated & 24 & 3.3 & 109 & 14.9 & 234 & 32.1 & 219 & 30.0 & 115 & 15.8 & 21 & 2.9 & 2 & 0.3 & 6 & 0.8 & 730 & 100.0 \\
\hline TOTAL & 3471 & 3.8 & 12686 & 13.9 & 24553 & 26.9 & 30785 & 33.7 & 16526 & 18.1 & 3073 & 3.4 & 163 & 0.2 & 58 & 0.1 & 91315 & 100.0 \\
\hline
\end{tabular}




\section{TABLE 21}

\begin{tabular}{|c|c|c|c|c|c|c|c|c|c|c|c|c|c|c|c|c|c|c|c|c|c|c|}
\hline \multirow{3}{*}{$\begin{array}{l}\text { Health } \\
\text { Area }\end{array}$} & \multicolumn{22}{|c|}{ Country of birth group } \\
\hline & \multicolumn{2}{|c|}{$\begin{array}{l}\text { English } \\
\text { speaking }\end{array}$} & \multicolumn{2}{|c|}{$\begin{array}{l}\text { Central } \\
\text { and South } \\
\text { America }\end{array}$} & \multicolumn{2}{|c|}{$\begin{array}{l}\text { Melanesia, } \\
\text { Micronesia, } \\
\text { and } \\
\text { Polynesia }\end{array}$} & \multicolumn{2}{|c|}{$\begin{array}{l}\text { Southern } \\
\text { Europe }\end{array}$} & \multicolumn{2}{|c|}{$\begin{array}{l}\text { Western } \\
\text { and } \\
\text { Northern } \\
\text { Europe }\end{array}$} & \multicolumn{2}{|c|}{$\begin{array}{c}\text { Eastern } \\
\text { Europe, } \\
\text { Russia, } \\
\text { Central } \\
\text { Asian and } \\
\text { Baltic } \\
\text { States }\end{array}$} & \multicolumn{2}{|c|}{$\begin{array}{l}\text { Middle } \\
\text { East and } \\
\text { Africa }\end{array}$} & \multicolumn{2}{|c|}{$\begin{array}{l}\text { South } \\
\text { East } \\
\text { Asia }\end{array}$} & \multicolumn{2}{|c|}{$\begin{array}{c}\text { North } \\
\text { East } \\
\text { Asia }\end{array}$} & \multicolumn{2}{|c|}{$\begin{array}{l}\text { Southern } \\
\text { Asia }\end{array}$} & \multicolumn{2}{|c|}{ TOTAL } \\
\hline & No. & $\%$ & No. & $\%$ & No. & $\%$ & No. & $\%$ & No. & $\%$ & No. & $\%$ & No. & $\%$ & No. & $\%$ & No. & $\%$ & No. & $\%$ & No. & $\%$ \\
\hline $\begin{array}{l}\text { Sydney South } \\
\text { West }\end{array}$ & 12614 & 62.1 & 292 & 1.4 & 673 & 3.3 & 418 & 2.1 & 141 & 0.7 & 140 & 0.7 & 2030 & 10.0 & 2363 & 11.6 & 897 & 4.4 & 748 & 3.7 & 20316 & 100.0 \\
\hline $\begin{array}{l}\text { South Eastern } \\
\text { Sydney and } \\
\text { Illawarra }\end{array}$ & 11964 & 80.7 & 175 & 1.2 & 126 & 0.8 & 219 & 1.5 & 180 & 1.2 & 149 & 1.0 & 456 & 3.1 & 645 & 4.3 & 650 & 4.4 & 266 & 1.8 & 14830 & 100.0 \\
\hline Sydney West & 11833 & 69.0 & 115 & 0.7 & 533 & 3.1 & 138 & 0.8 & 74 & 0.4 & 114 & 0.7 & 1379 & 8.0 & 969 & 5.6 & 682 & 4.0 & 1323 & 7.7 & 17160 & 100.0 \\
\hline $\begin{array}{l}\text { Northern Sydney } \\
\text { and Central } \\
\text { Coast }\end{array}$ & 11639 & 82.6 & 137 & 1.0 & 94 & 0.7 & 92 & 0.7 & 210 & 1.5 & 126 & 0.9 & 226 & 1.6 & 470 & 3.3 & 774 & 5.5 & 321 & 2.3 & 14089 & 100.0 \\
\hline $\begin{array}{l}\text { Hunter and New } \\
\text { England }\end{array}$ & 10150 & 96.1 & 17 & 0.2 & 48 & 0.5 & 20 & 0.2 & 44 & 0.4 & 16 & 0.2 & 49 & 0.5 & 115 & 1.1 & 61 & 0.6 & 40 & 0.4 & 10560 & 100.0 \\
\hline North Coast & 4891 & 96.2 & 11 & 0.2 & 15 & 0.3 & 3 & 0.1 & 37 & 0.7 & 5 & 0.1 & 28 & 0.6 & 54 & 1.1 & 27 & 0.5 & 14 & 0.3 & 5085 & 100.0 \\
\hline Greater Southern & 3884 & 96.0 & 6 & 0.1 & 31 & 0.8 & 4 & 0.1 & 19 & 0.5 & 3 & 0.1 & 23 & 0.6 & 31 & 0.8 & 17 & 0.4 & 27 & 0.7 & 4045 & 100.0 \\
\hline Greater Western & 3911 & 97.4 & 5 & 0.1 & 9 & 0.2 & 4 & 0.1 & 11 & 0.3 & 7 & 0.2 & 16 & 0.4 & 29 & 0.7 & 11 & 0.3 & 12 & 0.3 & 4015 & 100.0 \\
\hline Other-not stated & 629 & 86.9 & 7 & 1.0 & 9 & 1.2 & 3 & 0.4 & 9 & 1.2 & 4 & 0.6 & 9 & 1.2 & 22 & 3.0 & 24 & 3.3 & 8 & 1.1 & 724 & 100.0 \\
\hline TOTAL & 71515 & 78.7 & 765 & 0.8 & 1538 & 1.7 & 901 & 1.0 & 725 & 0.8 & 564 & 0.6 & 4216 & 4.6 & 4698 & 5.2 & 3143 & 3.5 & 2759 & 3.0 & 90824 & 100.0 \\
\hline
\end{tabular}

\section{TABLE 22}

MATERNAL ABORIGINALITY AND HEALTH AREA OF RESIDENCE, NSW 2006

Health Area Aboriginality

\begin{tabular}{|c|c|c|c|c|c|c|c|c|}
\hline \multirow[t]{3}{*}{ Heätn Area } & \multicolumn{8}{|c|}{ Adorigindaty } \\
\hline & \multicolumn{2}{|c|}{$\begin{array}{c}\text { Aboriginal - } \\
\text { Torres Strait Islander }\end{array}$} & \multicolumn{2}{|c|}{$\begin{array}{l}\text { Non-Aboriginal - } \\
\text { Torres Strait Islander }\end{array}$} & \multicolumn{2}{|c|}{ Not Stated } & \multicolumn{2}{|c|}{ TOTAL } \\
\hline & No. & $\%$ & No. & $\%$ & No. & $\%$ & No. & $\%$ \\
\hline Sydney South West & 213 & 1.0 & 20112 & 98.5 & 101 & 0.5 & 20426 & 100.0 \\
\hline South Eastern Sydney and Illawarra & 228 & 1.5 & 14596 & 97.2 & 196 & 1.3 & 15020 & 100.0 \\
\hline Sydney West & 284 & 1.7 & 16893 & 98.2 & 33 & 0.2 & 17210 & 100.0 \\
\hline Northern Sydney and Central Coast & 105 & 0.7 & 13998 & 99.0 & 36 & 0.3 & 14139 & 100.0 \\
\hline Hunter and New England & 594 & 5.6 & 9969 & 94.2 & 16 & 0.2 & 10579 & 100.0 \\
\hline North Coast & 406 & 8.0 & 4668 & 91.5 & 26 & 0.5 & 5100 & 100.0 \\
\hline Greater Southern & 193 & 4.8 & 3841 & 94.6 & 25 & 0.6 & 4059 & 100.0 \\
\hline Greater Western & 561 & 13.8 & 3406 & 84.1 & 85 & 2.1 & 4052 & 100.0 \\
\hline Other - not stated & 27 & 3.7 & 693 & 94.9 & 10 & 1.4 & 730 & 100.0 \\
\hline TOTAL & 2611 & 2.9 & 88176 & 96.6 & 528 & 0.6 & 91315 & 100.0 \\
\hline
\end{tabular}




\section{Duration of pregnancy at first antenatal visit}

In 2006, 87.5 per cent of mothers commenced antenatal care prior to 20 weeks gestation. This percentage varied from 87.2 per cent in the Hunter and New England Area to 93.9 per cent in the Northern Sydney and Central Coast Area (Table 23).

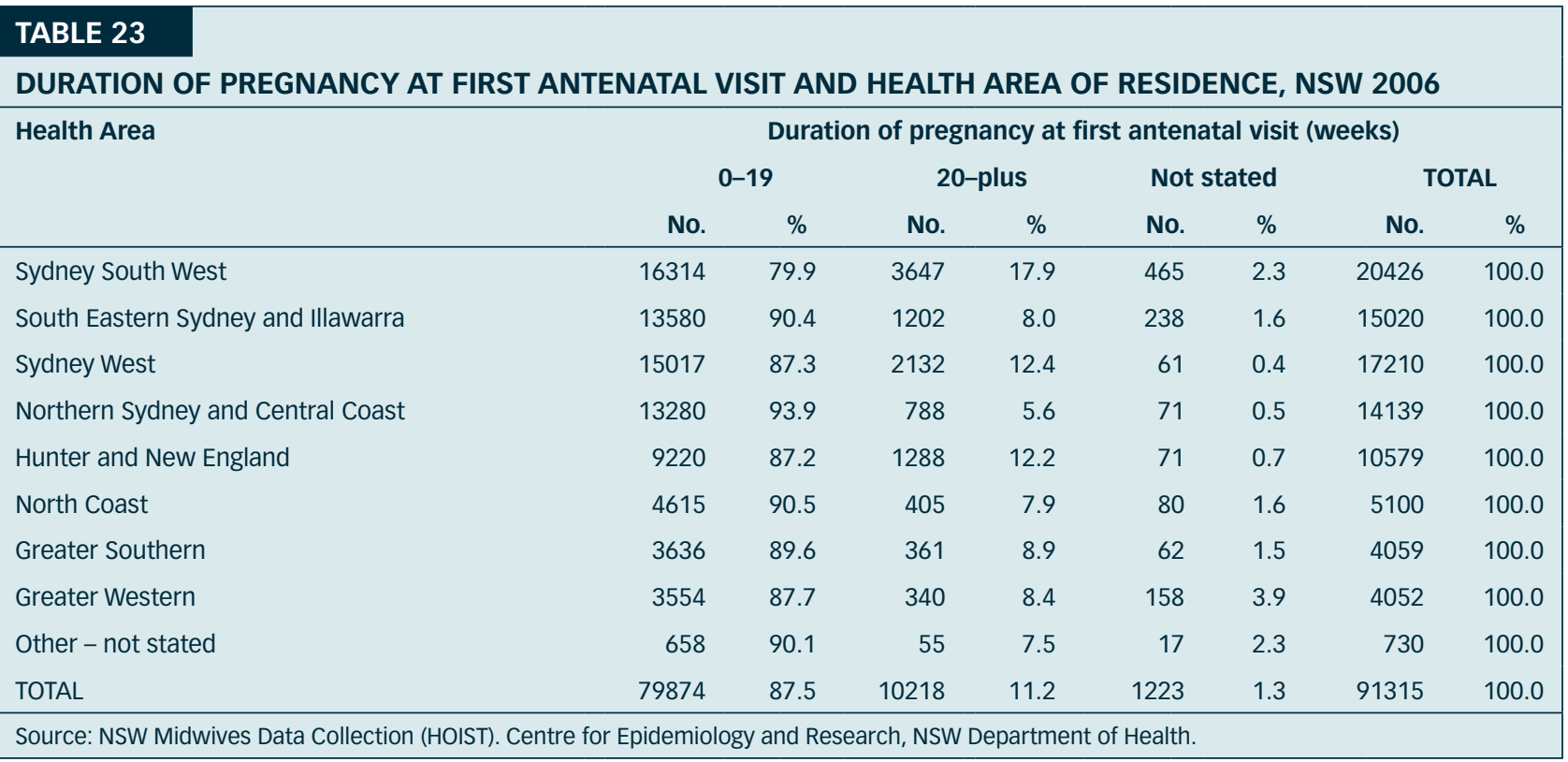

\section{Smoking in pregnancy}

In 2006, 12.8 per cent of mothers reported smoking in the second half of pregnancy (Table 24). The lowest reported rate was among mothers resident in the Northern
Sydney and Central Coast Area (6.8 per cent) and the highest rate among residents of the Greater Western Area (27.5 per cent).

\section{TABLE 24}

NUMBER OF CIGARETTES SMOKED IN THE SECOND HALF OF PREGNANCY, NSW 2006

Health Area Cigarettes smoked in the second half of pregnancy

\begin{tabular}{|c|c|c|c|c|c|c|c|c|c|c|c|c|}
\hline & \multicolumn{2}{|c|}{ None } & \multicolumn{2}{|c|}{ 1-10 per day } & \multicolumn{2}{|c|}{$\begin{array}{l}\text { More than } \\
10 \text { per day }\end{array}$} & \multicolumn{2}{|c|}{$\begin{array}{l}\text { Smoked amount } \\
\text { not stated }\end{array}$} & \multicolumn{2}{|c|}{ Not stated } & \multicolumn{2}{|c|}{ TOTAL } \\
\hline & No. & $\%$ & No. & $\%$ & No. & $\%$ & No. & $\%$ & No. & $\%$ & No. & $\%$ \\
\hline Sydney South West & 17280 & 84.6 & 1038 & 5.1 & 812 & 4.0 & 95 & 0.5 & 1201 & 5.9 & 20426 & 100.0 \\
\hline $\begin{array}{l}\text { South Eastern Sydney } \\
\text { and Illawarra }\end{array}$ & 13647 & 90.9 & 805 & 5.4 & 378 & 2.5 & 47 & 0.3 & 143 & 1.0 & 15020 & 100.0 \\
\hline Sydney West & 15078 & 87.6 & 1065 & 6.2 & 791 & 4.6 & 134 & 0.8 & 142 & 0.8 & 17210 & 100.0 \\
\hline $\begin{array}{l}\text { Northern Sydney and } \\
\text { Central Coast }\end{array}$ & 13091 & 92.6 & 562 & 4.0 & 352 & 2.5 & 49 & 0.3 & 85 & 0.6 & 14139 & 100.0 \\
\hline Hunter and New England & 8299 & 78.4 & 1201 & 11.4 & 998 & 9.4 & 72 & 0.7 & 9 & 0.1 & 10579 & 100.0 \\
\hline North Coast & 3929 & 77.0 & 593 & 11.6 & 520 & 10.2 & 40 & 0.8 & 18 & 0.4 & 5100 & 100.0 \\
\hline Greater Southern & 3115 & 76.7 & 477 & 11.8 & 442 & 10.9 & 9 & 0.2 & 16 & 0.4 & 4059 & 100.0 \\
\hline Greater Western & 2894 & 71.4 & 440 & 10.9 & 669 & 16.5 & 5 & 0.1 & 44 & 1.1 & 4052 & 100.0 \\
\hline Other - not stated & 596 & 81.6 & 62 & 8.5 & 54 & 7.4 & 4 & 0.5 & 14 & 1.9 & 730 & 100.0 \\
\hline TOTAL & 77929 & 85.3 & 6243 & 6.8 & 5016 & 5.5 & 455 & 0.5 & 1672 & 1.8 & 91315 & 100.0 \\
\hline
\end{tabular}




\section{Place of birth}

Ninety-six per cent of mothers chose to deliver in a hospital birthing suite in 2006, compared to 3.4 per cent who planned a birth centre birth and 0.2 per cent who planned a home birth (Table 25). Planned birth centre births were most commonly reported in the South Eastern Sydney and Illawarra, Sydney West and Sydney South West Areas.

\section{Labour}

In 2006, the onset of labour was spontaneous in 58.2 per cent of confinements (Table 26). Labour was induced in 24.7 per cent of confinements and no labour (elective caesarean section) was reported in 17.0 per cent. The rate of spontaneous onset of labour was highest among residents of the North Coast Area (65.6 per cent). The highest rate of induction of labour was among residents of the Sydney West Area (27.7 per cent).

\section{TABLE 25}

\section{PLACE OF BIRTH AND HEALTH AREA OF RESIDENCE, NSW 2006}

\begin{tabular}{|c|c|c|c|c|c|c|c|c|c|c|c|c|c|c|c|c|}
\hline \multirow[t]{3}{*}{ Health Area } & \multicolumn{16}{|c|}{ Place of birth } \\
\hline & \multicolumn{2}{|c|}{ Hospital } & \multicolumn{2}{|c|}{$\begin{array}{l}\text { Birth } \\
\text { Centre }\end{array}$} & \multicolumn{2}{|c|}{$\begin{array}{l}\text { Planned } \\
\text { birth centre/ } \\
\text { hospital } \\
\text { admission }\end{array}$} & \multicolumn{2}{|c|}{$\begin{array}{l}\text { Planned } \\
\text { homebirth }\end{array}$} & \multicolumn{2}{|c|}{$\begin{array}{l}\text { Planned } \\
\text { homebirth } \\
\text { / hospital } \\
\text { admission }\end{array}$} & \multicolumn{2}{|c|}{$\begin{array}{l}\text { Born } \\
\text { before } \\
\text { arrival }\end{array}$} & \multicolumn{2}{|c|}{ Not stated } & \multicolumn{2}{|c|}{ TOTAL } \\
\hline & No. & $\%$ & No. & $\%$ & No. & $\%$ & No. & $\%$ & No. & $\%$ & No. & $\%$ & No. & $\%$ & No. & $\%$ \\
\hline Sydney South West & 19999 & 97.9 & 223 & 1.1 & 98 & 0.5 & 11 & 0.1 & 6 & 0.0 & 79 & 0.4 & 10 & 0.0 & 20426 & 100.0 \\
\hline $\begin{array}{l}\text { South Eastern } \\
\text { Sydney and } \\
\text { Illawarra }\end{array}$ & 14151 & 94.2 & 425 & 2.8 & 349 & 2.3 & 15 & 0.1 & 12 & 0.1 & 44 & 0.3 & 24 & 0.2 & 15020 & 100.0 \\
\hline Sydney West & 16268 & 94.5 & 404 & 2.3 & 444 & 2.6 & 12 & 0.1 & 6 & 0.0 & 74 & 0.4 & 2 & 0.0 & 17210 & 100.0 \\
\hline $\begin{array}{l}\text { Northern Sydney and } \\
\text { Central Coast }\end{array}$ & 13875 & 98.1 & 149 & 1.1 & 53 & 0.4 & 12 & 0.1 & 1 & 0.0 & 46 & 0.3 & 3 & 0.0 & 14139 & 100.0 \\
\hline $\begin{array}{l}\text { Hunter and New } \\
\text { England }\end{array}$ & 9675 & 91.5 & 652 & 6.2 & 181 & 1.7 & 6 & 0.1 & 3 & 0.0 & 62 & 0.6 & 0 & 0.0 & 10579 & 100.0 \\
\hline North Coast & 4963 & 97.3 & 10 & 0.2 & 43 & 0.8 & 36 & 0.7 & 9 & 0.2 & 36 & 0.7 & 3 & 0.1 & 5100 & 100.0 \\
\hline Greater Southern & 3982 & 98.1 & 5 & 0.1 & 38 & 0.9 & 11 & 0.3 & 2 & 0.0 & 20 & 0.5 & 1 & 0.0 & 4059 & 100.0 \\
\hline Greater Western & 3949 & 97.5 & 7 & 0.2 & 29 & 0.7 & 7 & 0.2 & 6 & 0.1 & 27 & 0.7 & 27 & 0.7 & 4052 & 100.0 \\
\hline Other - not stated & 705 & 96.6 & 3 & 0.4 & 5 & 0.7 & 15 & 2.1 & 2 & 0.3 & 0 & 0.0 & 0 & 0.0 & 730 & 100.0 \\
\hline TOTAL & 87567 & 95.9 & 1878 & 2.1 & 1240 & 1.4 & 125 & 0.1 & 47 & 0.1 & 388 & 0.4 & 70 & 0.1 & 91315 & 100.0 \\
\hline
\end{tabular}

Source: NSW Midwives Data Collection (HOIST). Centre for Epidemiology and Research, NSW Department of Health.

\section{TABLE 26}

ONSET AND AUGMENTATION OF LABOUR AND HEALTH AREA OF RESIDENCE, NSW 2006

Health Area Onset of labour

\begin{tabular}{|c|c|c|c|c|c|c|c|c|c|c|c|c|c|c|c|c|c|c|c|c|}
\hline & \multicolumn{2}{|c|}{ Spontaneous } & \multicolumn{2}{|c|}{$\begin{array}{c}\text { Spontaneous } \\
\text { augmented } \\
\text { with ARM }\end{array}$} & \multicolumn{2}{|c|}{$\begin{array}{l}\text { Spontaneous } \\
\text { augmented } \\
\text { with oxytocics - } \\
\text { prostaglandins\# }\end{array}$} & \multicolumn{2}{|c|}{ No labour } & \multicolumn{2}{|c|}{$\begin{array}{l}\text { Induced } \\
\text { oxytocics/ } \\
\text { prosta- } \\
\text { glandins }\end{array}$} & \multicolumn{2}{|c|}{$\begin{array}{l}\text { Induced - } \\
\text { ARM only }\end{array}$} & \multicolumn{2}{|c|}{$\begin{array}{l}\text { Induced - } \\
\text { ARM + } \\
\text { oxytocics/ } \\
\text { prostaglandins }\end{array}$} & \multicolumn{2}{|c|}{$\begin{array}{l}\text { Induced - } \\
\text { other\#\# }\end{array}$} & \multicolumn{2}{|c|}{$\begin{array}{c}\text { Not } \\
\text { stated }\end{array}$} & \multicolumn{2}{|c|}{ TOTAL } \\
\hline & No. & $\%$ & No. & $\%$ & No. & $\%$ & No. & $\%$ & No. & $\%$ & No. & $\%$ & No. & $\%$ & No. & $\%$ & No. & $\%$ & No. & $\%$ \\
\hline Sydney South West & 9434 & 46.2 & 1022 & 5.0 & 2120 & 10.4 & 3214 & 15.7 & 1598 & 7.8 & 231 & 1.1 & 2674 & 13.1 & 122 & 0.6 & 11 & 0.1 & 20426 & 100.0 \\
\hline $\begin{array}{l}\text { South Eastern } \\
\text { Sydney and } \\
\text { Illawarra }\end{array}$ & 5981 & 39.8 & 1055 & 7.0 & 1399 & 9.3 & 2771 & 18.4 & 1059 & 7.1 & 238 & 1.6 & 2393 & 15.9 & 98 & 0.7 & 26 & 0.2 & 15020 & 100.0 \\
\hline Sydney West & 7007 & 40.7 & 1179 & 6.9 & 1439 & 8.4 & 2810 & 16.3 & 1415 & 8.2 & 253 & 1.5 & 3051 & 17.7 & 54 & 0.3 & 2 & 0.0 & 17210 & 100.0 \\
\hline $\begin{array}{l}\text { Northern Sydney } \\
\text { and Central Coast }\end{array}$ & 5611 & 39.7 & 821 & 5.8 & 1289 & 9.1 & 2973 & 21.0 & 1044 & 7.4 & 215 & 1.5 & 2140 & 15.1 & 43 & 0.3 & 3 & 0.0 & 14139 & 100.0 \\
\hline $\begin{array}{l}\text { Hunter and New } \\
\text { England }\end{array}$ & 4651 & 44.0 & 687 & 6.5 & 880 & 8.3 & 1570 & 14.8 & 863 & 8.2 & 234 & 2.2 & 1666 & 15.7 & 28 & 0.3 & 0 & 0.0 & 10579 & 100.0 \\
\hline North Coast & 2312 & 45.3 & 616 & 12.1 & 418 & 8.2 & 720 & 14.1 & 430 & 8.4 & 70 & 1.4 & 516 & 10.1 & 13 & 0.3 & 5 & 0.1 & 5100 & 100.0 \\
\hline Greater Southern & 1785 & 44.0 & 412 & 10.2 & 268 & 6.6 & 641 & 15.8 & 471 & 11.6 & 95 & 2.3 & 366 & 9.0 & 15 & 0.4 & 6 & 0.1 & 4059 & 100.0 \\
\hline Greater Western & 1609 & 39.7 & 465 & 11.5 & 259 & 6.4 & 710 & 17.5 & 368 & 9.1 & 104 & 2.6 & 481 & 11.9 & 39 & 1.0 & 17 & 0.4 & 4052 & 100.0 \\
\hline Other - not stated & 316 & 43.3 & 61 & 8.4 & 74 & 10.1 & 107 & 14.7 & 58 & 7.9 & 7 & 1.0 & 102 & 14.0 & 5 & 0.7 & 0 & 0.0 & 730 & 100.0 \\
\hline TOTAL & 38706 & 42.4 & 6318 & 6.9 & 8146 & 8.9 & 17.0 & 7306 & 8.0 & 1447 & 1.6 & 13389 & 14.7 & 417 & 0.5 & 70 & 0.1 & 91315 & 100.0 & 100.0 \\
\hline
\end{tabular}




\section{Birth}

Sixty per cent of confinements were by normal vaginal birth, 10.1 per cent were instrumental and 28.8 per cent were by caesarean section (Table 27). The highest rate of normal vaginal birth was among residents of the North Coast Area (69.5 per cent), while the highest rates of instrumental birth were among residents of South Eastern Sydney and Illawarra Area (13.4 per cent). The caesarean section rate varied from 24.6 per cent among mothers resident in the North Coast Area to 34.5 per cent in the Northern Sydney and Central Coast Area.

\section{Birth weight}

In 2006, 6.4 per cent of births were low birth weight (less than 2,500 grams). These comprised 0.7 per cent of birth weight less than 1,000 grams, 0.6 per cent in the 1,000 to 1,499 gram range and 5.1 per cent in the 1,500 to 2,499 gram range (Table 28). Rates of low birth weight ranged from 4.7 per cent in Greater Southern Area to 7.5 per cent in the Hunter and New England Area.

\section{TABLE 27}

\section{TYPE OF BIRTH BY HEALTH AREA OF RESIDENCE, NSW 2006}

\begin{tabular}{|c|c|c|c|c|c|c|c|c|c|c|c|c|c|c|c|c|}
\hline \multirow[t]{3}{*}{ Health Area } & \multicolumn{16}{|c|}{ Place of birth } \\
\hline & \multicolumn{2}{|c|}{$\begin{array}{l}\text { Normal } \\
\text { vaginal }\end{array}$} & \multicolumn{2}{|c|}{ Forceps } & \multicolumn{2}{|c|}{$\begin{array}{l}\text { Vacuum } \\
\text { extraction }\end{array}$} & \multicolumn{2}{|c|}{$\begin{array}{l}\text { Vaginal } \\
\text { breech }\end{array}$} & \multicolumn{2}{|c|}{$\begin{array}{c}\text { Elective } \\
\text { caesarean } \\
\text { section }\end{array}$} & \multicolumn{2}{|c|}{$\begin{array}{c}\text { Emergency } \\
\text { caesarean } \\
\text { section\# }\end{array}$} & \multicolumn{2}{|c|}{ Not stated } & \multicolumn{2}{|c|}{ TOTAL } \\
\hline & No. & $\%$ & No. & $\%$ & No. & $\%$ & No. & $\%$ & No. & $\%$ & No. & $\%$ & No. & $\%$ & No. & $\%$ \\
\hline Sydney South West & 12985 & 63.6 & 482 & 2.4 & 1490 & 7.3 & 84 & 0.4 & 3209 & 15.7 & 2140 & 10.5 & 36 & 0.2 & 20426 & 100.0 \\
\hline $\begin{array}{l}\text { South Eastern } \\
\text { Sydney and } \\
\text { Illawarra }\end{array}$ & 8141 & 54.2 & 500 & 3.3 & 1515 & 10.1 & 61 & 0.4 & 2770 & 18.4 & 1967 & 13.1 & 66 & 0.4 & 15020 & 100.0 \\
\hline Sydney West & 10628 & 61.8 & 681 & 4.0 & 874 & 5.1 & 57 & 0.3 & 2807 & 16.3 & 2151 & 12.5 & 12 & 0.1 & 17210 & 100.0 \\
\hline $\begin{array}{l}\text { Northern Sydney } \\
\text { and Central Coast }\end{array}$ & 7480 & 52.9 & 518 & 3.7 & 1212 & 8.6 & 36 & 0.3 & 2973 & 21.0 & 1904 & 13.5 & 16 & 0.1 & 14139 & 100.0 \\
\hline $\begin{array}{l}\text { Hunter and New } \\
\text { England }\end{array}$ & 6811 & 64.4 & 367 & 3.5 & 626 & 5.9 & 57 & 0.5 & 1570 & 14.8 & 1140 & 10.8 & 8 & 0.1 & 10579 & 100.0 \\
\hline North Coast & 3515 & 68.9 & 103 & 2.0 & 181 & 3.5 & 30 & 0.6 & 720 & 14.1 & 535 & 10.5 & 16 & 0.3 & 5100 & 100.0 \\
\hline Greater Southern & 2576 & 63.5 & 145 & 3.6 & 232 & 5.7 & 7 & 0.2 & 641 & 15.8 & 448 & 11.0 & 10 & 0.2 & 4059 & 100.0 \\
\hline Greater Western & 2569 & 63.4 & 75 & 1.9 & 170 & 4.2 & 29 & 0.7 & 710 & 17.5 & 458 & 11.3 & 41 & 1.0 & 4052 & 100.0 \\
\hline Other - not stated & 482 & 66.0 & 14 & 1.9 & 32 & 4.4 & 5 & 0.7 & 107 & 14.7 & 84 & 11.5 & 6 & 0.8 & 730 & 100.0 \\
\hline TOTAL & 55187 & 60.4 & 2885 & 3.2 & 6332 & 6.9 & 366 & 0.4 & 15507 & 17.0 & 10827 & 11.9 & 211 & 0.2 & 91315 & 100.0 \\
\hline
\end{tabular}

Source: NSW Midwives Data Collection (HOIST). Centre for Epidemiology and Research, NSW Department of Health.

\# Emergency caesarean section includes caesarean section where the onset of labour was not stated.

\section{TABLE 28}

\section{BIRTH WEIGHT AND HEALTH AREA OF RESIDENCE, NSW 2006}

Health Area

\begin{tabular}{|c|c|c|c|c|c|c|c|c|c|c|c|c|c|c|c|c|c|c|c|c|c|c|}
\hline & \multicolumn{2}{|c|}{$\begin{array}{c}\text { Less } \\
\text { than } 500\end{array}$} & \multicolumn{2}{|c|}{$500-999$} & \multicolumn{2}{|c|}{$\begin{array}{l}1000- \\
1499\end{array}$} & \multicolumn{2}{|c|}{$\begin{array}{c}1500- \\
1999\end{array}$} & \multicolumn{2}{|c|}{$\begin{array}{c}2000- \\
2499\end{array}$} & \multicolumn{2}{|c|}{$\begin{array}{c}2500- \\
2999\end{array}$} & \multicolumn{2}{|c|}{$\begin{array}{c}3000- \\
3499\end{array}$} & \multicolumn{2}{|c|}{$\begin{array}{c}2500- \\
3999\end{array}$} & \multicolumn{2}{|c|}{$\begin{array}{r}4000- \\
4499\end{array}$} & \multicolumn{2}{|c|}{$4500+$} & \multicolumn{2}{|c|}{ TOTAL } \\
\hline & No. & $\%$ & No. & $\%$ & No. & $\%$ & No. & $\%$ & No. & $\%$ & No. & $\%$ & No. & $\%$ & No. & $\%$ & No. & $\%$ & No. & $\%$ & No. & $\%$ \\
\hline $\begin{array}{l}\text { Sydney South } \\
\text { West }\end{array}$ & 53 & 0.3 & 90 & 0.4 & 134 & 0.6 & 275 & 1.3 & 820 & 4.0 & 3384 & 16.3 & 7605 & 36.7 & 6195 & 29.9 & 1856 & 8.9 & 300 & 1.4 & 20750 & 100.0 \\
\hline $\begin{array}{l}\text { South Eastern } \\
\text { Sydney and } \\
\text { Illawarra }\end{array}$ & 33 & 0.2 & 75 & 0.5 & 100 & 0.7 & 186 & 1.2 & 581 & 3.8 & 2245 & 14.7 & 5521 & 36.1 & 4800 & 31.4 & 1489 & 9.7 & 232 & 1.5 & 15285 & 100.0 \\
\hline Sydney West & 51 & 0.3 & 85 & 0.5 & 125 & 0.7 & 217 & 1.2 & 670 & 3.8 & 2704 & 15.5 & 6375 & 36.4 & 5240 & 30.0 & 1706 & 9.8 & 306 & 1.7 & 17491 & 100.0 \\
\hline $\begin{array}{l}\text { Northern Sydney } \\
\text { and Central Coast }\end{array}$ & 33 & 0.2 & 53 & 0.4 & 71 & 0.5 & 180 & 1.3 & 464 & 3.2 & 1893 & 13.2 & 5167 & 35.9 & 4684 & 32.6 & 1568 & 10.9 & 263 & 1.8 & 14384 & 100.0 \\
\hline $\begin{array}{l}\text { Hunter and New } \\
\text { England }\end{array}$ & 36 & 0.3 & 49 & 0.5 & 63 & 0.6 & 168 & 1.6 & 454 & 4.2 & 1587 & 14.8 & 3557 & 33.1 & 3333 & 31.0 & 1218 & 11.3 & 254 & 2.4 & 10735 & 100.0 \\
\hline North Coast & 14 & 0.3 & 14 & 0.3 & 23 & 0.4 & 45 & 0.9 & 207 & 4.0 & 786 & 15.2 & 1717 & 33.2 & 1657 & 32.0 & 590 & 11.4 & 117 & 2.3 & 5175 & 100.0 \\
\hline Greater Southern & 4 & 0.1 & 6 & 0.1 & 13 & 0.3 & 37 & 0.9 & 133 & 3.2 & 576 & 14.0 & 1409 & 34.3 & 1339 & 32.6 & 493 & 12.0 & 94 & 2.3 & 4111 & 100.0 \\
\hline Greater Western & 12 & 0.3 & 28 & 0.7 & 24 & 0.6 & 50 & 1.2 & 192 & 4.7 & 627 & 15.3 & 1453 & 35.4 & 1247 & 30.4 & 387 & 9.4 & 73 & 1.8 & 4107 & 100.0 \\
\hline $\begin{array}{l}\text { Other - not } \\
\text { stated }\end{array}$ & 3 & 0.4 & 11 & 1.5 & 9 & 1.2 & 9 & 1.2 & 33 & 4.4 & 104 & 14.0 & 270 & 36.3 & 210 & 28.3 & 82 & 11.0 & 10 & 1.3 & 743 & 100.0 \\
\hline TOTAL & 239 & 0.3 & 411 & 0.4 & 562 & 0.6 & 1167 & 1.3 & 3554 & 3.8 & 13906 & 15.0 & 33074 & 35.6 & 28705 & 30.9 & 9389 & 10.1 & 1649 & 1.8 & 92781 & 100.0 \\
\hline
\end{tabular}




\section{Gestational age}

The majority of births (91.0 per cent) were at term, and 1.6 per cent were post-term (42-plus weeks). The 7.4 per cent of preterm births comprised 0.7 per cent born at less than 28 weeks, 0.7 per cent at 28-31 weeks, and 5.9 per cent at 32-36 weeks. The highest rate of preterm birth was in the Hunter and New England Area (8.9 per cent), while the lowest was 5.4 per cent in the Greater Southern Area (Table 29).

\section{TABLE 29}

\begin{tabular}{|c|c|c|c|c|c|c|c|c|c|c|c|c|c|c|}
\hline \multirow[t]{3}{*}{ Health Area } & \multicolumn{14}{|c|}{ Gestational age (weeks) } \\
\hline & \multicolumn{2}{|c|}{$<28$} & \multicolumn{2}{|c|}{$28-31$} & \multicolumn{2}{|c|}{$32-36$} & \multicolumn{2}{|c|}{$37-41$} & \multicolumn{2}{|c|}{$42+$} & \multicolumn{2}{|c|}{ Not stated } & \multicolumn{2}{|c|}{ TOTAL } \\
\hline & No. & $\%$ & No. & $\%$ & No. & $\%$ & No. & $\%$ & No. & $\%$ & No. & $\%$ & No. & $\%$ \\
\hline Sydney South West & 141 & 0.7 & 144 & 0.7 & 1194 & 5.8 & 18850 & 90.8 & 410 & 2.0 & 11 & 0.1 & 20750 & 100.0 \\
\hline $\begin{array}{l}\text { South Eastern Sydney } \\
\text { and Illawarra }\end{array}$ & 119 & 0.8 & 122 & 0.8 & 901 & 5.9 & 13787 & 90.2 & 355 & 2.3 & 1 & 0.0 & 15285 & 100.0 \\
\hline Sydney West & 136 & 0.8 & 132 & 0.8 & 1052 & 6.0 & 16024 & 91.6 & 145 & 0.8 & 2 & 0.0 & 17491 & 100.0 \\
\hline $\begin{array}{l}\text { Northern Sydney and } \\
\text { Central Coast }\end{array}$ & 87 & 0.6 & 94 & 0.7 & 776 & 5.4 & 13196 & 91.7 & 229 & 1.6 & 2 & 0.0 & 14384 & 100.0 \\
\hline $\begin{array}{l}\text { Hunter and New } \\
\text { England }\end{array}$ & 86 & 0.8 & 102 & 1.0 & 767 & 7.1 & 9593 & 89.4 & 187 & 1.7 & 0 & 0.0 & 10735 & 100.0 \\
\hline North Coast & 31 & 0.6 & 29 & 0.6 & 311 & 6.0 & 4734 & 91.5 & 69 & 1.3 & 1 & 0.0 & 5175 & 100.0 \\
\hline Greater Southern & 17 & 0.4 & 11 & 0.3 & 195 & 4.7 & 3825 & 93.0 & 63 & 1.5 & 0 & 0.0 & 4111 & 100.0 \\
\hline Greater Western & 44 & 1.1 & 28 & 0.7 & 249 & 6.1 & 3751 & 91.3 & 33 & 0.8 & 2 & 0.0 & 4107 & 100.0 \\
\hline Other - not stated & 24 & 3.3 & 109 & 14.9 & 234 & 32.1 & 219 & 30.0 & 115 & 15.8 & 6 & 0.8 & 730 & 100.0 \\
\hline TOTAL & 3471 & 3.8 & 12686 & 13.9 & 24553 & 26.9 & 30785 & 33.7 & 16526 & 18.1 & 58 & 0.1 & 91315 & 100.0 \\
\hline
\end{tabular}

\section{Infant feeding}

In 2006, infant feeding on discharge from hospital was reported for 36,481 live births. The majority of babies (74.8 per cent) were reported to be fully breast-fed, 12.3 per cent were having infant formula only, and a further 2.1 per cent were having a combination of breast-feeding and infant formula. Rates of full breast-feeding varied from 65.0 per cent in the Sydney South West Area to 85.7 per cent in the Northern Sydney and Central Coast Area.

\section{TABLE 30}

LIVE BIRTHS BY INFANT FEEDING ON DISCHARGE AND HEALTH AREA OF RESIDENCE, NSW 2006

Health Area

\begin{tabular}{|c|c|c|c|c|c|c|c|c|c|c|c|c|c|c|}
\hline & \multicolumn{2}{|c|}{$\begin{array}{l}\text { Breast } \\
\text { feeding } \\
\text { only }\end{array}$} & \multicolumn{2}{|c|}{$\begin{array}{l}\text { Expressed } \\
\text { breast milk } \\
\text { only }\end{array}$} & \multicolumn{2}{|c|}{$\begin{array}{l}\text { Infant } \\
\text { formula } \\
\text { only }\end{array}$} & \multicolumn{2}{|c|}{$\begin{array}{l}\text { Breast-feeding } \\
\text { and infant } \\
\text { formula }\end{array}$} & \multicolumn{2}{|c|}{$\begin{array}{c}\text { Other } \\
\text { combination }\end{array}$} & \multicolumn{2}{|c|}{ Not stated } & \multicolumn{2}{|c|}{ TOTAL } \\
\hline & No. & $\%$ & No. & $\%$ & No. & $\%$ & No. & $\%$ & No. & $\%$ & No. & $\%$ & No. & $\%$ \\
\hline Sydney South West & 2636 & 65.0 & 48 & 1.2 & 586 & 14.5 & 197 & 4.9 & 129 & 3.2 & 458 & 11.3 & 4054 & 100.0 \\
\hline $\begin{array}{l}\text { South Eastern Sydney } \\
\text { and Illawarra }\end{array}$ & 5478 & 77.1 & 168 & 2.4 & 954 & 13.4 & 151 & 2.1 & 176 & 2.5 & 182 & 2.6 & 7109 & 100.0 \\
\hline Sydney West & 2590 & 60.0 & 42 & 1.0 & 431 & 10.0 & 84 & 1.9 & 129 & 3.0 & 1038 & 24.1 & 4314 & 100.0 \\
\hline $\begin{array}{l}\text { Northern Sydney and } \\
\text { Central Coast }\end{array}$ & 3783 & 85.7 & 29 & 0.7 & 224 & 5.1 & 67 & 1.5 & 101 & 2.3 & 208 & 4.7 & 4412 & 100.0 \\
\hline $\begin{array}{l}\text { Hunter and New } \\
\text { England }\end{array}$ & 2241 & 73.6 & 37 & 1.2 & 419 & 13.8 & 67 & 2.2 & 55 & 1.8 & 225 & 7.4 & 3044 & 100.0 \\
\hline North Coast & 4164 & 82.8 & 44 & 0.9 & 497 & 9.9 & 68 & 1.4 & 95 & 1.9 & 159 & 3.2 & 5027 & 100.0 \\
\hline Greater Southern & 3005 & 75.7 & 35 & 0.9 & 657 & 16.5 & 88 & 2.2 & 67 & 1.7 & 118 & 3.0 & 3970 & 100.0 \\
\hline Greater Western & 2863 & 73.2 & 27 & 0.7 & 653 & 16.7 & 48 & 1.2 & 37 & 0.9 & 283 & 7.2 & 3911 & 100.0 \\
\hline Other - not stated & 524 & 81.9 & 10 & 1.6 & 74 & 11.6 & 2 & 0.3 & 10 & 1.6 & 20 & 3.1 & 640 & 100.0 \\
\hline TOTAL & 27284 & 74.8 & 440 & 1.2 & 4495 & 12.3 & 772 & 2.1 & 799 & 2.2 & 2691 & 7.4 & 36481 & 100.0 \\
\hline
\end{tabular}




\section{Perinatal outcomes}

The perinatal mortality rate in 2006 was 8.8 per 1,000 births. This includes all births and deaths of babies of at least 400 grams birth weight or at least 20 weeks gestation
(Table 31). The rate varied from 6.3 per 1,000 in the Greater Southern Area to 10.5 per 1,000 in the Greater Western Area.

\section{TABLE 31}

PERINATAL MORTALITY BY HEALTH AREA OF RESIDENCE, NSW 2006\#

Health Area Perinatal outcome

\begin{tabular}{|c|c|c|c|c|c|c|c|c|c|c|c|c|c|}
\hline & \multicolumn{2}{|c|}{$\begin{array}{l}\text { Liveborn } \\
\text { surviving }\end{array}$} & \multicolumn{2}{|c|}{ Stillborn } & \multicolumn{2}{|c|}{$\begin{array}{l}\text { Neonatal } \\
\text { death }\end{array}$} & \multicolumn{2}{|c|}{ Not stated } & \multicolumn{2}{|c|}{$\begin{array}{l}\text { Death - age } \\
\text { not stated }\end{array}$} & \multicolumn{2}{|c|}{ Total births } & $\begin{array}{l}\text { Perinatal } \\
\text { mortality / } \\
1000 \text { births }\end{array}$ \\
\hline & No. & $\%$ & No. & $\%$ & No. & $\%$ & No. & $\%$ & No. & $\%$ & No. & $\%$ & No. \\
\hline Sydney South West & 20527 & 98.9 & 129 & 0.6 & 59 & 0.3 & 35 & 0.2 & 0 & 0.0 & 20750 & 100.0 & 9.1 \\
\hline $\begin{array}{l}\text { South Eastern Sydney } \\
\text { and Illawarra }\end{array}$ & 15129 & 99.0 & 112 & 0.7 & 36 & 0.2 & 7 & 0.0 & 1 & 0.0 & 15285 & 100.0 & 9.7 \\
\hline Sydney West & 17307 & 98.9 & 119 & 0.7 & 34 & 0.2 & 31 & 0.2 & 0 & 0.0 & 17491 & 100.0 & 8.7 \\
\hline $\begin{array}{l}\text { Northern Sydney and } \\
\text { Central Coast }\end{array}$ & 14277 & 99.3 & 72 & 0.5 & 30 & 0.2 & 5 & 0.0 & 0 & 0.0 & 14384 & 100.0 & 7.1 \\
\hline $\begin{array}{l}\text { Hunter and New } \\
\text { England }\end{array}$ & 10630 & 99.0 & 75 & 0.7 & 30 & 0.3 & 0 & 0.0 & 0 & 0.0 & 10735 & 100.0 & 9.8 \\
\hline North Coast & 5120 & 98.9 & 37 & 0.7 & 9 & 0.2 & 7 & 0.1 & 2 & 0.0 & 5175 & 100.0 & 8.9 \\
\hline Greater Southern & 4083 & 99.3 & 18 & 0.4 & 8 & 0.2 & 1 & 0.0 & 1 & 0.0 & 4111 & 100.0 & 6.3 \\
\hline Greater Western & 4036 & 98.3 & 25 & 0.6 & 18 & 0.4 & 26 & 0.6 & 2 & 0.0 & 4107 & 100.0 & 10.5 \\
\hline Other - not stated & 731 & 98.4 & 5 & 0.7 & 5 & 0.7 & 2 & 0.3 & 0 & 0.0 & 743 & 100.0 & - \\
\hline TOTAL & 91840 & 99.0 & 592 & 0.6 & 229 & 0.2 & 114 & 0.1 & 6 & 0.0 & 92781 & 100.0 & 8.8 \\
\hline
\end{tabular}

Source: NSW Midwives Data Collection (HOIST). Centre for Epidemiology and Research, NSW Department of Health.

\# Perinatal deaths include deaths reported to the MDC only. As the MDC form is completed at discharge or transfer of the baby; deaths occurring

after this time may not be reported to the MDC. 


\section{TABLE 32}

LIVE BIRTHS BY HEALTH AREA AND STATISTICAL LOCAL AREA OF RESIDENCE, NSW 2006

\begin{tabular}{|c|c|c|c|c|c|}
\hline Health Area - Statistical Local Area & No. & $\%$ & Health Area - Statistical Local Area & No. & $\%$ \\
\hline Sydney South West & & & Sutherland Shire - East & 1325 & 8.7 \\
\hline Ashfield & 565 & 2.7 & Sutherland Shire - West & 1524 & 10.0 \\
\hline Bankstown - North-East & 1226 & 6.0 & Sydney - Inner & 152 & 1.0 \\
\hline Bankstown - North-West & 972 & 4.7 & Sydney - South & 156 & 1.0 \\
\hline Bankstown - South & 721 & 3.5 & Sydney - East & 417 & 2.7 \\
\hline Burwood & 309 & 1.5 & Waverley & 975 & 6.4 \\
\hline Camden & 817 & 4.0 & Wollongong - Inner & 1186 & 7.8 \\
\hline Campbelltown - North & 1203 & 5.8 & Wollongong - Balance & 1221 & 8.1 \\
\hline Campbelltown - South & 1094 & 5.3 & Woollahra & 737 & 4.9 \\
\hline Canada Bay - Concord & 439 & 2.1 & TOTAL & 15166 & 100.0 \\
\hline Canada Bay - Drummoyne & 572 & 2.8 & Northern Sydney and Central Coast & & \\
\hline Canterbury & 2334 & 11.3 & Gosford - East & 706 & 4.9 \\
\hline Fairfield - East & 1739 & 8.4 & Gosford - West & 1276 & 8.9 \\
\hline Fairfield - West & 889 & 4.3 & Hornsby - North & 789 & 5.5 \\
\hline Leichhardt & 1020 & 5.0 & Hornsby - South & 1107 & 7.7 \\
\hline Liverpool - East & 1889 & 9.2 & Hunters Hill & 169 & 1.2 \\
\hline Liverpool - West & 1161 & 5.6 & Ku-ring-gai & 899 & 6.3 \\
\hline Marrickville & 1229 & 6.0 & Lane Cove & 488 & 3.4 \\
\hline Strathfield & 347 & 1.7 & Manly & 587 & 4.1 \\
\hline Sydney - Inner & 3 & 0.0 & Mosman & 373 & 2.6 \\
\hline Sydney - South & 540 & 2.6 & North Sydney & 895 & 6.3 \\
\hline Sydney - West & 390 & 1.9 & Pittwater & 690 & 4.8 \\
\hline Wingecarribee & 455 & 2.2 & Ryde & 1265 & 8.8 \\
\hline Wollondilly & 672 & 3.3 & Warringah & 2092 & 14.6 \\
\hline TOTAL & 20586 & 100.0 & Willoughby & 1035 & 7.2 \\
\hline Sydney West & & & Wyong - North-East & 1019 & 7.1 \\
\hline Auburn & 1246 & 7.2 & Wyong - South and West & 917 & 6.4 \\
\hline Baulkham Hills - Central & 935 & 5.4 & TOTAL & 14307 & 100.0 \\
\hline Baulkham Hills - North & 767 & 4.4 & Hunter and New England & & \\
\hline Baulkham Hills - South & 443 & 2.6 & Armidale Dumaresq - City & 227 & 2.1 \\
\hline Blacktown - North & 1609 & 9.3 & Armidale Dumaresq - Balance & 99 & 0.9 \\
\hline Blacktown - South-East & 1559 & 9.0 & Cessnock & 671 & 6.3 \\
\hline Blacktown - South-West & 1787 & 10.3 & Dungog & 78 & 0.7 \\
\hline Blue Mountains & 910 & 5.2 & Glen Innes Severn (A) & 115 & 1.1 \\
\hline Hawkesbury & 934 & 5.4 & Gloucester & 51 & 0.5 \\
\hline Holroyd & 1683 & 9.7 & Greater Taree & 489 & 4.6 \\
\hline Lithgow & 243 & 1.4 & Great Lakes & 338 & 3.2 \\
\hline Parramatta - Inner & 672 & 3.9 & Gunnedah & 155 & 1.5 \\
\hline Parramatta - North-East & 514 & 3.0 & Guyra & 53 & 0.5 \\
\hline Parramatta - North-West & 526 & 3.0 & Gwydir (A) & 67 & 0.6 \\
\hline Parramatta - South & 646 & 3.7 & Inverell - Pt A & 71 & 0.7 \\
\hline Penrith - East & 1558 & 9.0 & Inverell - Pt B & 154 & 1.4 \\
\hline Penrith - West & 1309 & 7.5 & Lake Macquarie - East & 692 & 6.5 \\
\hline TOTAL & 17341 & 100.0 & Lake Macquarie - North & 978 & 9.2 \\
\hline South Eastern Sydney and Illawarra & & & Lake Macquarie - West & 544 & 5.1 \\
\hline Botany & 538 & 3.5 & Liverpool Plains (A) & 81 & 0.8 \\
\hline Hurstville & 1006 & 6.6 & Maitland & 975 & 9.1 \\
\hline Kiama & 187 & 1.2 & Moree Plains & 199 & 1.9 \\
\hline Kogarah & 708 & 4.7 & Muswellbrook & 277 & 2.6 \\
\hline Randwick & 1726 & 11.4 & Narrabri & 184 & 1.7 \\
\hline Rockdale & 1438 & 9.5 & Newcastle - Inner City & 667 & 6.3 \\
\hline Shellharbour & 816 & 5.4 & Newcastle - Outer West & 604 & 5.7 \\
\hline Shoalhaven - Pt A & 398 & 2.6 & Newcastle - Throsby & 720 & 6.8 \\
\hline Shoalhaven - Pt B & 656 & 4.3 & Port Stephens & 731 & 6.9 \\
\hline
\end{tabular}


TABLE 32 (continued)

LIVE BIRTHS BY HEALTH AREA AND STATISTICAL LOCAL AREA OF RESIDENCE, NSW 2006

\begin{tabular}{|c|c|c|c|c|c|}
\hline Health Area - Statistical Local Area & No. & $\%$ & Health Area - Statistical Local Area & No. & $\%$ \\
\hline Singleton & 353 & 3.3 & Lockhart & 31 & 0.8 \\
\hline Tamworth Regional - Pt A & 566 & 5.3 & Murray & 9 & 0.2 \\
\hline Tamworth Regional - Pt B & 189 & 1.8 & Murrumbidgee & 27 & 0.7 \\
\hline Tenterfield & 36 & 0.3 & Narrandera & 97 & 2.4 \\
\hline Upper Hunter Shire & 201 & 1.9 & Palerang - Pt A & 28 & 0.7 \\
\hline Uralla & 55 & 0.5 & Palerang - Pt B & 8 & 0.2 \\
\hline Walcha & 40 & 0.4 & Queanbeyan & 215 & 5.3 \\
\hline TOTAL & 10660 & 100.0 & Snowy River & 75 & 1.8 \\
\hline North Coast & & & Temora & 66 & 1.6 \\
\hline Ballina & 376 & 7.3 & Tumbarumba & 19 & 0.5 \\
\hline Bellingen & 130 & 2.5 & Tumut & 125 & 3.1 \\
\hline Byron & 277 & 5.4 & Upper Lachlan & 88 & 2.2 \\
\hline Clarence Valley - Coast & 167 & 3.3 & Urana & 10 & 0.2 \\
\hline Clarence Valley - Grafton & 273 & 5.3 & Wagga Wagga - Pt A & 757 & 18.5 \\
\hline Clarence Valley - Balance & 97 & 1.9 & Wagga Wagga - Pt B & 112 & 2.7 \\
\hline Coffs Harbour - Pt A & 601 & 11.7 & Wakool & 7 & 0.2 \\
\hline Coffs Harbour - Pt B & 159 & 3.1 & Yass Valley (A) & 14 & 0.3 \\
\hline Hastings - Pt A & 442 & 8.6 & Young & 145 & 3.5 \\
\hline Hastings - Pt B & 304 & 5.9 & Other & 2 & 0.0 \\
\hline Kempsey & 375 & 7.3 & TOTAL & 4092 & 100.0 \\
\hline Kyogle & 86 & 1.7 & Greater Western & & \\
\hline Lismore - Pt A & 393 & 7.7 & Bathurst Regional - Pt A & 409 & 10.1 \\
\hline Lismore - Pt B & 184 & 3.6 & Bathurst Regional - Pt B & 48 & 1.2 \\
\hline Nambucca & 210 & 4.1 & Blayney - Pts A and B & 86 & 2.1 \\
\hline Richmond Valley - Casino & 129 & 2.5 & Bogan & 66 & 1.6 \\
\hline Richmond Valley - Balance & 162 & 3.2 & Bourke & 49 & 1.2 \\
\hline Tweed - Tweed-Heads & 452 & 8.8 & Brewarrina & 27 & 0.7 \\
\hline Tweed - Tweed Coast & 90 & 1.8 & Broken Hill & 217 & 5.4 \\
\hline Tweed - Pt B & 224 & 4.4 & Cabonne - Pts A, B and C & 128 & 3.2 \\
\hline TOTAL & 5131 & 100.0 & Central Darling & 18 & 0.4 \\
\hline Greater Southern & & & Cobar & 92 & 2.3 \\
\hline Albury & 7 & 0.2 & Coonamble & 69 & 1.7 \\
\hline Bega Valley & 287 & 7.0 & Cowra & 143 & 3.5 \\
\hline Berrigan & 21 & 0.5 & Dubbo - Pt A & 610 & 15.0 \\
\hline Bland & 74 & 1.8 & Dubbo - Pt B & 59 & 1.5 \\
\hline Bom - Balance & 26 & 0.6 & Forbes & 117 & 2.9 \\
\hline Boorowa & 16 & 0.4 & Gilgandra & 71 & 1.8 \\
\hline Carrathool & 39 & 1.0 & Lachlan & 86 & 2.1 \\
\hline Conargo & 20 & 0.5 & Mid-Western Regional - Pt A & 261 & 6.4 \\
\hline Coolamon & 49 & 1.2 & Mid-Western Regional - Pt B & 35 & 0.9 \\
\hline Cooma-Monaro & 84 & 2.1 & Narromine & 107 & 2.6 \\
\hline Cootamundra & 82 & 2.0 & Oberon & 126 & 3.1 \\
\hline Deniliquin & 103 & 2.5 & Orange & 598 & 14.7 \\
\hline Eurobodalla & 383 & 9.4 & Parkes & 225 & 5.5 \\
\hline Greater Argyle - Goulburn & 255 & 6.2 & Walgett & 105 & 2.6 \\
\hline Greater Argyle - Balance & 29 & 0.7 & Warren & 31 & 0.8 \\
\hline Greater Hume Shire - Pt B & 27 & 0.7 & Warrumbungle Shire & 110 & 2.7 \\
\hline Griffith & 366 & 8.9 & Weddin & 48 & 1.2 \\
\hline Gundagai & 44 & 1.1 & Wellington & 106 & 2.6 \\
\hline Harden & 33 & 0.8 & Wentworth & 5 & 0.1 \\
\hline Hay & 48 & 1.2 & Other & 4 & 0.1 \\
\hline Jerilderie & 13 & 0.3 & TOTAL & 4056 & 100.0 \\
\hline Junee & 79 & 1.9 & Other - Not stated & 736 & 100.0 \\
\hline Leeton & 172 & 4.2 & TOTAL NSW & 92075 & 100.0 \\
\hline
\end{tabular}

